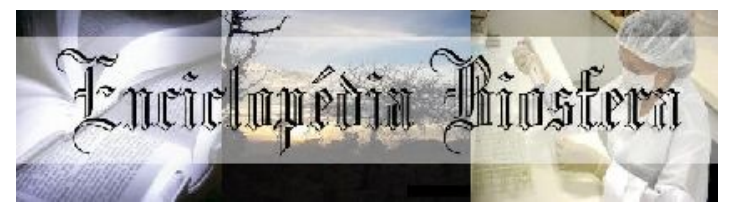

\title{
ESTUDO DA VIABILIDADE DO USO DE PROTOTIPAGEM RÁPIDA 3D EM PROCESSOS PRODUTIVOS NO MEIO RURAL
}

$\underline{\text { Rafael Zucca }}{ }^{1}$, Rodrigo Couto Santos ${ }^{2}$, Juliano Lovatto ${ }^{3}$, Felipo Lovatto ${ }^{4}$ Arthur Carniato Sanches ${ }^{5}$

1. Engenheiro Agrícola, Mestrando em Engenharia Agrícola, Faculdade de Ciências

Agrárias, UFGD, Dourados-MS, Brasil (rafael-zucca@hotmail.com).Dourados-Brasil;

2. Engenheiro Agrícola, Professor Doutor da Faculdade de Ciências Agrárias, UFGD;

3. Engenheiro Civil, Mestrando em Engenharia Agrícola da Faculdade de Ciências Agrárias, UFGD;

4. Arquiteto, Mestrando em Engenharia Agrícola da Faculdade de Ciências Agrárias, UFGD.

5. Agrônomo, Professor Doutor da Faculdade de Ciências Agrárias, UFGD.

Recebido em: 22/09/2018 - Aprovado em: 23/11/2018 - Publicado em: 03/12/2018

DOI: 10.18677/EnciBio_2018B45

\begin{abstract}
RESUMO
As impressoras 3D quando surgiram eram utilizadas apenas por grandes industrias, em função de seu custo muito elevado. Porém, estão surgindo novos modelos e com valores mais acessíveis, proporcionando um aumento na aquisição e o uso para diferentes aplicações de tal tecnologia, seja por empresas ou consumidores entusiastas em conhecer esta ferramenta tecnológica emergente. O emprego de impressão 3D, está acarretando uma transformação na forma de criação de produtos e a maneira em que são produzidos, tornando-se uma tecnologia cada vez emergente e inovadora, sendo possível reproduzir com eficiência produtos impressos individualmente, com a qualidade e o acabamento de grandes indústrias de produção em massa. Deste modo, a impressão tridimensional possibilita a criação de peças ou objetos de acordo com a necessidade do usuário ou setor de produção, possibilitando a confecção de objetos para fins comerciais, peças de reposição de variados processos produtivos, outras impressoras 3D, entre outros produtos. Posto isto, este trabalho teve por objetivo, analisar como a nova tecnologia vem sendo incorporada ao desenvolvimento de produtos e o impacto de sua aplicação no meio rural, demonstrando fomas de utilização da impressão 3D com finalidades diversas, conceituando a possibilidade de prototipagem de peças de reposição e fabricação de protótipos comerciais, mostrando como cada indivíduo, empresa ou propriedade rural, pode aplicar conceitos de impressão 3D na fabricação de peças ou produtos, variando o tamanho e formato, especificação e quantidade exigida, tudo conforme a necessidade e demanda de seu processo produtivo.
\end{abstract}

PALAVRAS-CHAVE: Impressoras 3D, Processos produtivos, Tecnologias agrícolas.

\section{STUDY OF VIABILITY OF THE USE OF RAPID 3D-TYPING IN PRODUCTIVE PROCESSES IN THE RURAL ENVIRONMENT}

\begin{abstract}
The 3D printers when they appeared were used only by large industries, due to its very high cost. However, new and more affordable models are emerging, providing an increase in the acquisition and use for different applications of such technology,
\end{abstract}


either by companies or consumers enthusiastic about this emerging technological tool. The use of 3D printing is bringing about a transformation in the form of product creation and the way in which it is produced, becoming an emerging and innovative technology, being able to efficiently reproduce printed products individually, with quality and finishing of large mass-production industries. Thus, three-dimensional printing allows the creation of parts or objects according to the user's needs or production sector, making objects for commercial purposes, spare parts of various production processes, other 3D printers, among other products. The aim of this work was to analyze how the new technology has been incorporated into product development and the impact of its application in rural areas, demonstrating the use of 3D printing for different purposes, conceptualizing, the possibility of prototyping of spare parts and manufacturing of commercial prototypes, showing how each individual, company or property can apply 3D printing concepts in manufacturing parts or products, varying the size and format, specification and quantity required, all according to the need and demand of its productive process.

KEYWORDS: 3D printing, Productive Process, Agriculture Technology.

\section{INTRODUÇÃO}

A era industrial 4.0 tem impactado significativamente o modo de vida da população e a forma como ocorre o progresso da sociedade, sendo que, a história do desenvolvimento humano está diretamente relacionada ao avanço tecnológico (JIANG et al., 2017).

De acordo com Santos et al. (2015), a globalização mundial vem acarretando ao setor agropecuário diversas mudanças em decorrência do aumento da concorrência e de consumidores cada vez mais exigentes. Dessa forma, aumentou o investimento do produtor no seu processo produtivo, com a finalidade de suprir as grandes demandas do mercado mundial.

Segundo afirmam Zucca et al. (2018) há no setor agrícola uma demanda por infraestrutura e avanços tecnológicos nos próximos anos, no qual existe espaço para o uso de materiais sustentáveis e novas tecnologias que visem o aumento da produtividade. Assim, as atividades rurais atualmente fazem uso de várias tecnologias na busca de melhorar a produtividade, procurando sempre melhorias para o uso dos recursos disponíveis evitando dispêndio de energia e tempo durante o processo produtivo, englobando desde maquinários, sistemas de administração e automação, entre outros.

O potencial da impressão 3D para transformar o modelo de produção atual está se tornando evidente, baseado em sua capacidade de criar dispositivos, materiais e estruturas personalizadas e com geometrias complexas. O método está em constante aperfeiçoamento, havendo uma crescente variedade de materiais de impressão disponíveis, funcionais, simples ou compostos, que seria dificil de se obter com o uso de técnicas de fabricação tradicionais (KALSOOM et al., 2018).

Dessa forma, a impressão tridimensional surge como uma alternativa para aumento da produtividade no meio rural, justificando o presente estudo, buscando novas técnologias para diminuir o dispêndio de tempo na aquisição de peças de reposição, tendo em vista que várias propriedades são isoladas ou distantes de cidades e grandes centros. Possibilitando a confecção de peças obsoletas que quando quebram acarretam a parada de máquinas, impactando diretamente no processo produtivo e a viabiliadade de se criar protótipos de peças e equipamentos em menor tempo e sem sair da propriedade ou ter que contratar serviços de 
terceiros (SCOTT, 2016).

Posto isto, este trabalho teve por objetivo, abordar novas tecnologias advindas da indústria 4.0 e o impacto que podem causar no meio rural, focando na tecnologia de prototipagem 3D e a forma com que a mesma pode ser aplicada em processos produtivos agrícolas, conceituando a tecnologia e a possibilidade de impressão de peças, ferramentas, objetos, entre outros produtos, demonstrando as aplicações para cada setor e os métodos de impressão 3D utilizados na fabricação de peças e produtos, variando o tamanho, a especificação e quantidade exigida, conforme a necessidade e demanda.

Realizou-se uma revisão sistemática da literatura, envolvendo referências do banco de dados da Capes, livros, dissertações, relatórios anuais, referências da base de dados científicos da SciELO (Scientific Eletronic Library Online), Science Direct, Taylor \& Francis Library, Oxford Academy e Wiley Online Library buscando os artigos mais relevantes publicados até o ano de 2018.

Dessa maneira, a metodologia consistiu em uma pesquisa bibliográfica sobre os avanços tecnológicos na agricultura, insdutria 4.0, impressoras 3D, métodos de impressão 3D e seu surgimento, serviços que utilizam a prototipagem 3D em seus produtos.

\section{DESENVOLVIMENTO}

\section{Avanço de novas tecnologias e o crescimento da prototipagem 3D}

O avanço tecnológico implica em profundas mudanças em quase todos os aspectos da vida humana e no progresso da sociedade, acarretando uma melhor qualidade de vida e um maior nível de inteligência humana. Acelerar o avanço tecnológico é de interesse de todas as sociedades, sendo uma questão ainda mais crítica em economias emergentes em que a aplicação da tecnologia geralmente está atrasada se comparada às economias já estabelecidas (JIANG et al., 2017).

De acordo com Nikander (2017), o avanço tecnológico ocorre em vários setores das sociedades modernas, por exemplo, no uso de energias renováveis, biotecnologia, Internet, telecomunicações, etc. As Tecnologias da Informação e da Comunicação (TIC), de sensoriamento remoto, instrumentação avançada, automação e robótica indicam que as tecnologias ganharão cada vez mais espaço nas propriedades brasileiras no futuro. Sendo que, esses processos e ferramentas permitirão o uso de recursos naturais da forma mais inteligente e sustentável, alcançando mais produtividade, eficiência na produção e garantindo a sustentabilidade (BRASIL, 2012).

A impressão 3D é uma das tecnologias emergentes, assim sua utilização é consequência do grau de avanços tecnológicos no mundo e o surgimento de novas tecnologias. Segundo Wohlers (2016), a indústria 3D cresceu para um valor estimado de cinco bilhões de dólares durante 2015 com estimativa para ultrapassar os 20 bilhões de dólares em 2020, conforme Figura 1, implicando em um crescimento do mercado de aproximadamente $25,9 \%$ em tal ano, enquanto no ano anterior atingiu crescimento de 34,9\%, a maior taxa durante os últimos 17 anos. 


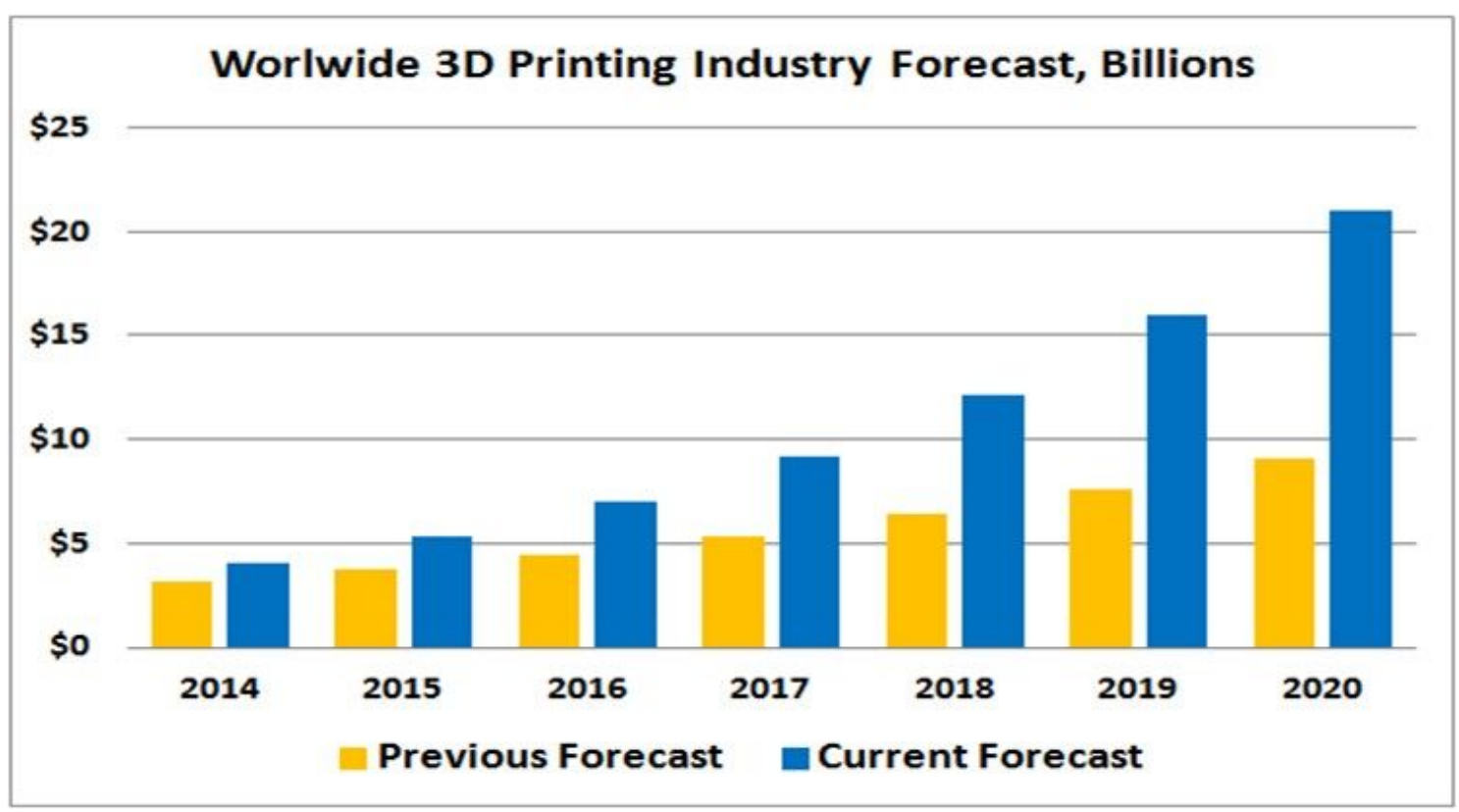

FIGURA 1. Revisão das previsões para o mercado de 3DP em 2014.

Fonte: Wohlers (2014)

Considerando outro fator na avaliação do crescimento da impressão 3D nos últimos anos, o número de usuários que existem no mercado no que diz respeito a produção de sistemas industriais de 3D, levando em consideração que em 2011 tinha-se 31 empresas produtoras de impressoras 3D, esse número saltou para 62 em 2015, evidenciando que o valor duplicou em apenas cinco anos. Sobre as impressoras Desktop, estima-se que o crescimento passou de 160 mil unidades vendidas no ano de 2014, para 278 mil unidades em 2015, conforme Figura 2 (WOHLERS, 2016).

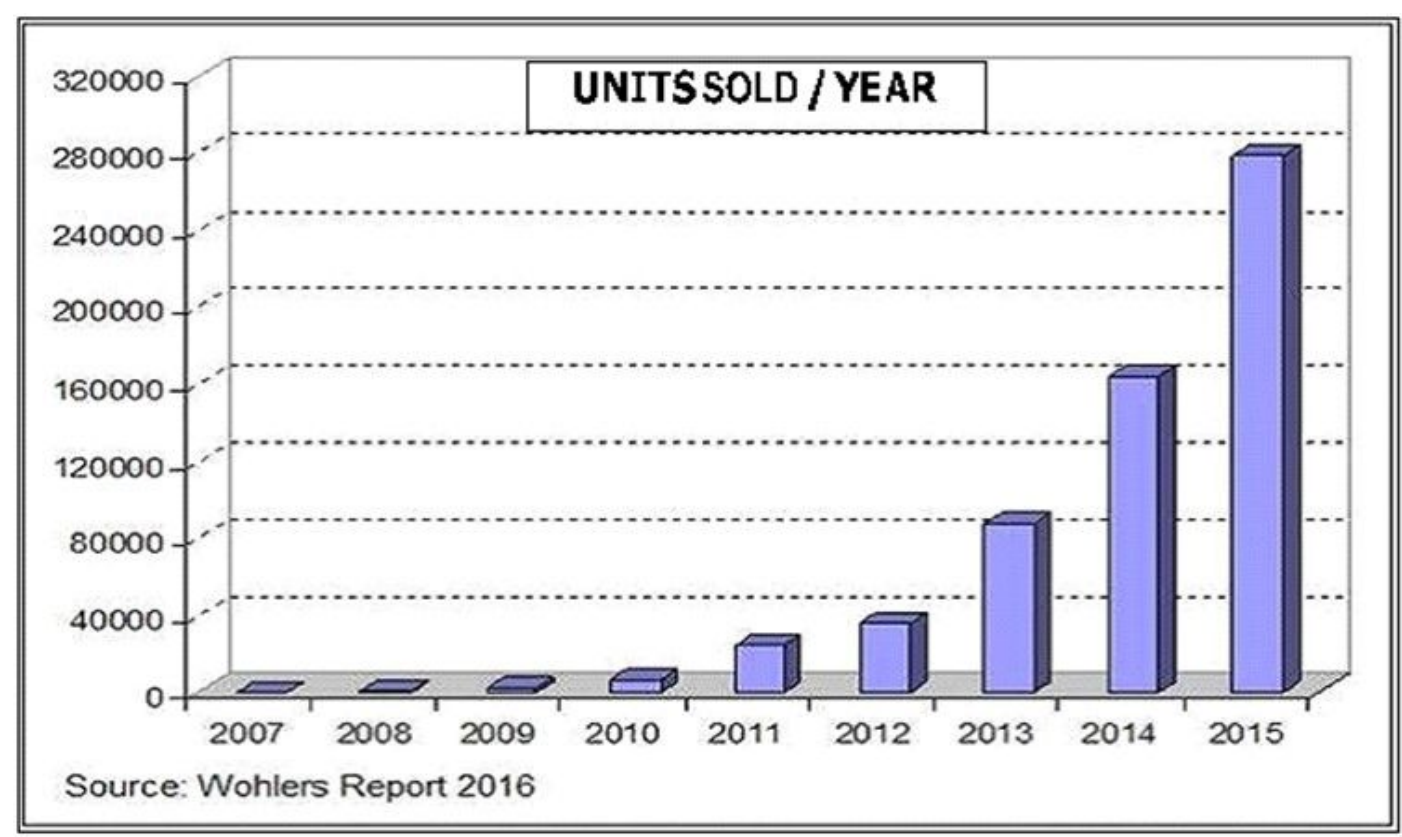

FIGURA 2. Evolução nas vendas de impressoras 3D individuais. Fonte: Wohlers (2016)

Ainda de acordo com o relatório da empresa americana de consultoria 
Wohlers (2017), considerado como o mais reconhecido e prestigiado relatório sobre AM - Additive Manufacturing do mundo, os negócios com impressoras 3D realizaram no mundo em 2016 uma movimentação de 16,8 bilhões de reais, contribuindo com a evolução de $30 \%$ se comparada com 2015 . Estima-se que até 2020 , os valores cheguem a 21 bilhões de reais e a estimativa era que, em 2016, existiam no mundo 455,7 mil unidades de impressão 3D, número que deve saltar a 6,7 milhões em 2020 , sendo que o mercado brasileiro representa apenas $2 \%$ do total de negócios mundial. Estima-se que até 2020, a prototipagem será o principal uso em dispositivos de fabricação utilizados por fabricantes. Nesse ano, quase $65 \%$ dos fabricantes irão utilizar a impressão 3D para produzirem componentes dos produtos que vendem.

\section{Industria 4.0}

Schwab (2017) afirma que no início do seculo XXI, com o desenvolvimento da internet, sensores cada vez mais pequenos e potentes, softwares e hardwares cada vez mais sofisticados, e a capacidade das máquinas em aprenderem e colaborarem criando redes de "coisas", teve inicio uma transformação na indústria, com um impacto na sociedade, economia e na competitividade que poderá transformar o mundo da forma como é conhecido surgindo assim o conceito de indústria 4.0.

Os termos Indústria 4.0, Smart Factory, Intelegent Factory, Factory of the future, descrevem como serão as fábricas no futuro (BAYGIN et al., 2016). Serão fábricas mais inteligentes, dinâmicas, flexíveis e ágeis, que fabricam produtos inteligentes, com equipamentos inteligentes e em cadeias produtivas inteligentes (HUBA; KOZÁK, 2016).

De acordo com Da Costa (2017), a indústria está em transformação a uma velocidade nunca vista antes, sendo impulsionada pelo desenvolvimento e utilização de novas tecnologias, mais evoluídas e ágeis. Tal transformação é tão rápida que se fala em uma nova Revolução Industrial, a quarta, podendo provocar alterações profundas, na indústria, sociedade, economia, nos valores e na forma como serão escolhidos os produtos e serviços, tendo como base inovação colaborativa, manufatura aditiva, as plataformas digitais, entre outras.

O World Economic Forum - WEF lançou em 2015 um relatório intitulado "Deep Shift Technology Tipping Points and Societal Impact" ou Pontos de Ruptura da Tecnologia de Diferentes Tipos e Profundo Impacto Social em português, que teve como base uma consulta à 800 executivos e especialistas do setor de tecnologias da informação e comunicação, com finalidade de sugerir 21 pontos críticos de mudanças técnologicas para o futuro (WEF, 2015).

No relatório foram identificadas seis tendências que estão moldando a sociedade e criando oportunidades até o ano de 2025, sendo:

Pessoas e a internet: Redes sociais, o modo como interagem as pessoas;

Computadores, comunicações e armazenamento: Redução no custo, tamanho dos computadores e tecnologias de comunicação;

Internet das coisas: Sensores cada vez menores e baratos utilizados em casas, produtos, cidades, transportes e processos produtivos;

Inteligência Artificial e Big-Data: Crescimento exponencial da digitalização, softwares com algoritmos mais sofisticados e capazes de aprender e evoluir sozinhos, ocupando lugares antes reservados ao homem, incluindo lugares de tomada decisão;

Economia partilhada e confiança distribuída: Redes socias, 
compartilhamento de recursos em vez da aquisição, bitcoins que estão a criar modelos de negócio, reformulando os já existentes, modificando as formas de relações e a perceção de confiança em parcerias;

Digitalização da matéria: A impressão em 3D de objetos e peças utilizando materiais cada vez mais evoluídos e inteligentes, transformando a era industrial, referente a prototipagem, logística de distribuição e criando um modelo de oportunidades para as indústrias, mercado doméstico e saúde, entre outros inúmeros setores.

Os métodos da Indústria 4.0 estão sendo aplicados em diversas áreas da agricultura, resultando no surgimento de termos como Agricultura 4.0 e Fazenda 4.0, impactando diretamente nos modelos tradicionais de produção agrícola. Porém, aplicam-se mais no campo da agricultura de precisão e, em menor grau, para sistemas autônomos embora haja um enorme espaço de crescimento nesse campo, no que diz respeito a melhorias na cadeia de suprimentos no meio rural, na criação de peças e protótipos (BRAUN et al., 2018).

\section{Novas tecnologias no meio agrícola}

Com o término do século $\mathrm{XX}$, ocorreu uma revolução no setor do agronegócio mundial, utilizando-se cada vez mais de novas tecnologias para melhorias na produção rural (REDIVO et al., 2012). De acordo com Ramos (2015) compreendendo o processo de inovação como fenômeno econômico e como um diferencial competitivo, demonstrado como objetivos nas formas de gestões atuais.

$\mathrm{Na}$ busca em aprimorar e dar mais qualidade as práticas utilizadas na agricultura, a ciência e a tecnologia buscam desenvolver conhecimentos e novas técnicas capazes de modificarem completamente a agricultura (MENDONÇA; LEHFELD, 2015). Segundo Zylberstajn et al. (2014), os dados estatísticos comprovam ganhos de produtividade no meio rural brasileiro, principalmente em atividades de exportação, pois rompeu-se a barreira do desafio tecnológico.

Conforme apontam pesquisas desenvolvidas pela Emprapa (2014), a agropecuária tem como desafio continuar se desenvolvendo de forma sustentável, em tempos difíceis e de mudanças. Sendo necessárias tecnologias mais eficientes visando atender às grandes demandas de fibras, alimentos, energia entre várias outras matérias primas da indústria e de produção excedente para fins de exportação, o que garantirá o suprimento da demanda por alimentos e energética global.

O desenvolvimento tecnológico da agricultura brasileira deve ser abordado como um guia, indicando caminhos a serem seguidos, afim de alcançar futuramente o desenvolvimento de tecnologias a serem aplicadas na agropecuária brasileira. (EMBRAPA, 2014).

Observa-se um grande papel de destaque para processos que alavancam a produtividade, utilizando processos que englobam inovações organizacionais e novas tecnologias (FORNAZIER; VIEIRA FILHO, 2012). Tendo assim como uma alternativa, a aplicação de impressão $3 \mathrm{D}$ em processos produtivos no meiro rural.

De acordo com Low et al. (2017), a manufatura aditiva, mais conhecida como impressão 3D, está impactando as áreas de fabricação e prototipagem, fornecendo uma rápida alternativa e com baixo custo, para técnicas de fabricação mais tradicionais, como moldagem, prototipagem e usinagem através de Controle Numérico Computadorizado (CNC).

O potencial da impressão 3D está relacionado ao fato de que esta reduz o 
grande tempo perdido entre as mudanças de equipamentos e atualizações de equipamentes em uma linha de produção em larga escala, permitindo frequentes inovações em um item durante o processo de fabricação, e em contrapartida, aumenta o grau de personalização na produção em massa (KAPETANIOU et al., 2018).

As impressoras 3D possibilitam a fabricação de objetos tridimensionais com detalhes complexos, isso sem o criador necessitar de habilidades de manufatura e 0 uso de inúmeras ferramentas e recursos. Por essas características, estão sendo estudadas novas possibilidades de produção, e o impacto que isto pode trazer para a indústria, onde cada pessoa poderá desenvolver o seu próprio produto, ou então receber o arquivo digital para imprimir o produto em casa (GAO et al., 2015).

\section{Impressoras 3D}

As primeiras experiências com impressora 3D são da década de 70 , sendo que a indústria de impressão 3D teve início no final de 1980, tais impressoras tinham alto custo na época. A grande diferença entre este novo método, para o existente ao da época, é o fato de imprimirem camadas após camadas, no sentido de ser uma produção aditiva, o método existente era um método subtrativo, que retira o material para formar o desenho final (KIETZMANN et al., 2015).

Conforme afirmam Da Silva et al. (2018), o desenvolvimento do Comando Numérico Computadorizado (CNC) veio para automatizar determinados processos de fabricação, diminuindo elevados custos com mão de obra e tempo. As máquinas CNC controlam equipamentos utilizando programas de computadores, sem que haja necessidade de um operador. Além de controlarem impressoras 3D, o método CNC é utilizado também na confecção de placas de circuito impresso (PCI), sendo de extrema importância para a indústria eletroeletrônica, já que é a base para o funcionamento de inúmeros equipamentos.

De acordo com Berman (2012), as impressoras 3D são similares com as impressoras que imprimem textos e cópias de documentos, porém as impressoras comuns recebem um documento eletrônico gerado por um computador, já as impressoras 3D utilizando-se do modelo Comando Numérico Computadorizado (CNC), ou seja, são controladas por ferramentas programáveis por computadores, que utilizando comandos por códigos que controlam e executam os movimentos a serem realizados pela máquina.

Assim, a diferença basicamente é que a impressora 3D tem a capacidade de criar um objeto sólido tridimensional a partir de diversos tipos de materiais, tais objetos projetados utilizando programas de livre acesso (HAUSMAN; HORNE, 2014).

Conforme afirmam Hausman e Horne (2014), a tecnologia da impressão 3D teve um grande desenvolvimento, depois que o pesquisador britânico Adrian Bowyer criou o sistema RepRap, como sendo o primeiro tipo de prototipagem rápida de auto replicação, recuperando e reutilizando motores e materiais de uma loja de ferragem e com a possibilidade de imprimir vários componentes para fabricação de outras impressoras 3D.

O Engenheiro Mecânico Adrian Bowyer, em 2004 publicou online o primeiro documento sobre o projeto RepRap, sendo que a primeira impressora 3D proveniente de tal projeto foi montada no ano de 2007 , os projetos difundiram os conhecimentos para que outras pessoas interessadas pudessem montar sua própria impressora 3D, com um custo mais acessível do que as comercializadas (JONES et 
al., 2011).

Santos (2016) afirma que com o início da tecnologia na década de 80, nas décadas seguintes foram desenvolvidas novas tecnologias, além do método inicial de impressão através do sistema de estereolitografia, o método através da fusão de plástico. Há uma divisão na comercialização das impressoras 3D com vários modelos disponíveis, um segmento que busca maior qualidade e possibilidade de fabricar objetos complexos para a indústria, sendo este processo mais caro, e outra linha com objetivo de fazer objetos mais simples e protótipos para pequenas empresas com custo mais acessível, conforme Figura 3.

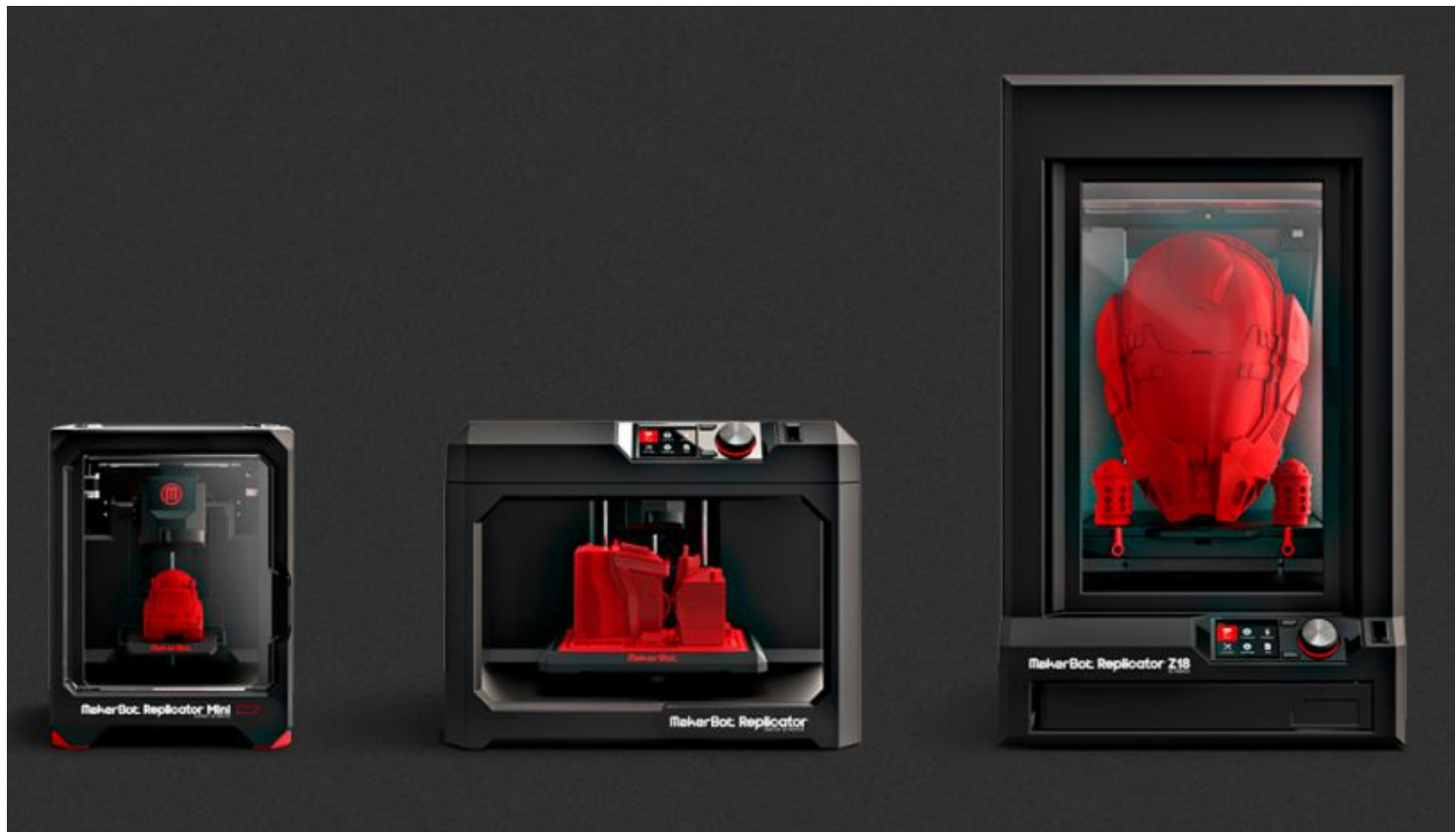

FIGURA 3. Modelos de impressoras 3D.

Fonte: HD Store (2017)

Assim, como visto na Figura 3, existem vários modelos de impressores 3D, com tamanhos e área de impressão diferentes, que utilizam diferentes tipos de materiais para impressão, variando o valor, umas com preços mais elevados e outras mais baratas, conforme a necessidade do usuário.

Encontram-se também disponíveis no mercado nacional ou importados de outros países, modelos mais simples e fáceis de operar, com preços mais acessíveis ao consumidor sem pouco conhecimento e que pretende se iniciar nessa tecnologia emergente, como mostra Figura 4. 


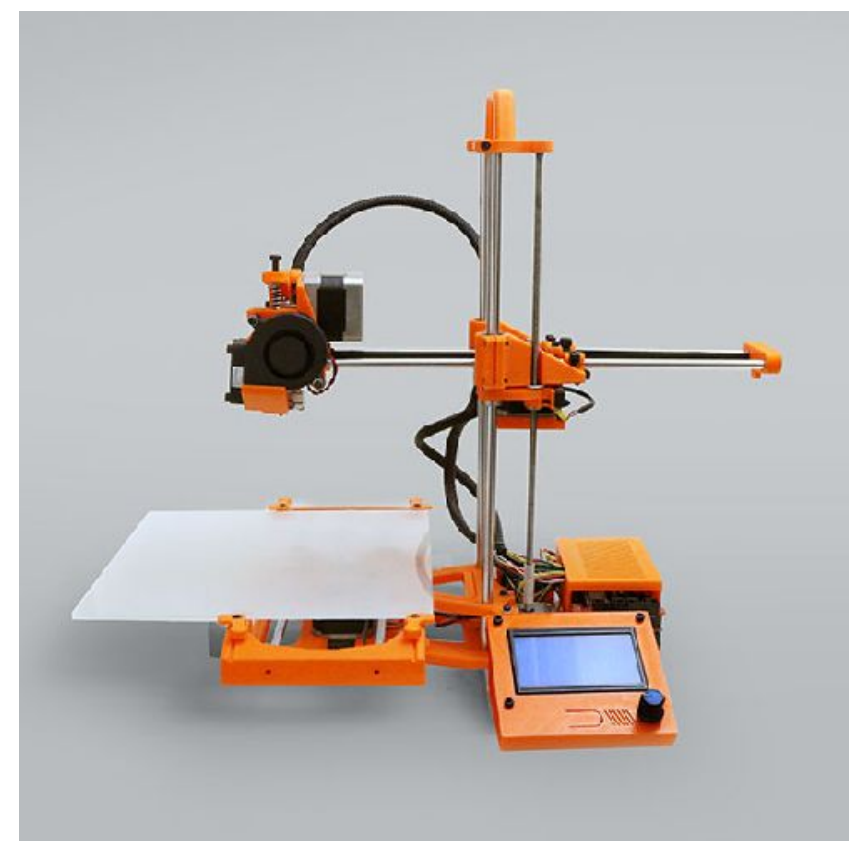

FIGURA 4. Impressora 3D, modelo Stella.

Fonte: HD Store (2017)

Há também um seguimento de impressoras 3D montadas a partir de projetos Open source disponibilizados em sites diversos, com projetos de baixo custo, como visto na Figura 5, que utilizam o método RepRap citado anteriormente por Hausman e Horne (2014), adotado pela cultura Maker para fabricar a impressora, sendo esta autorreplicável, onde são impressas partes de novas impressoras, e no caso da maioria dos componentes eletrônicos ou vem do reuso de descartes de outros equipamentos eletrônicos, como motores de passo ou são simplesmente comprados na forma de kits de peças vendidos a baixo custo no mercado especializado.

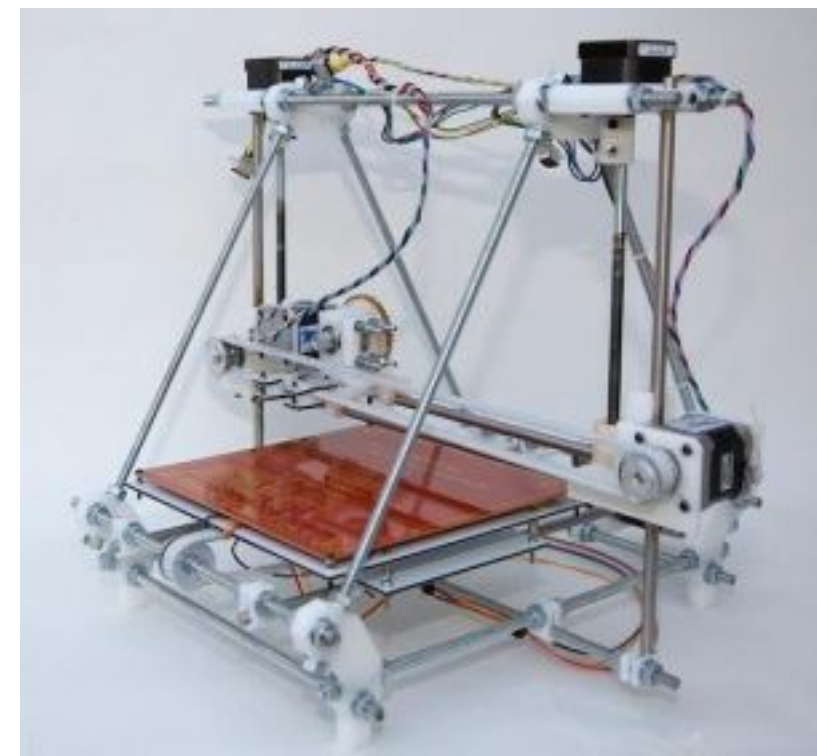

FIGURA 5. Modelo simples fabricado utilizando o método RepRap.

Métodos de Impressão 3D

Fonte: HD Store (2017)

No setor de impressão 3D através da técnica de manufatura aditiva existem 
vários métodos, estes variam de acordo com o material e tipo de técnica utilizada, como, por exemplo, a Estereolitografia (SL ou SLA), PolyJet, Tecnologia Zprinter e Modelação por Extrusão de Plástico (FDM), cada método apresenta propriedades únicas, que possibilitam vantagens e desvantagens e assim a escolha depende de onde será utilizado o sistema, existindo alguns parâmetros de comparação entre eles como velocidade de produção pretendida, durabilidade e quanto de trabalho de pós impressão será necessário para realizar o acabamento da peça (CONNER et al., 2014).

Segundo Abreu (2015), o método pioneiro da tecnologia é o Estereolitografia, que se baseia na foto polimerização de resinas líquidas pela incidência de um feixe laser de raios ultravioleta (UV), ou seja, o objeto é feito através de um raio que solidifica a resina dentro de um tanque, sendo direcionado através de um conjunto de espelhos. Após o processo de impressão é preciso colocar a peça em um forno com radiação Ultravioleta para cura. Assim, seus beneficios são que o processo oferece grande precisão no objeto, sem diferenças dimensionais e sem porosidade. Por um outro lado as desvantagens são um tempo elevado para fabricação do objeto, equipamentos caros e pouca resistência do material.

Outro tipo de técnica é a chamada PolyJet, mais recente, desenvolvida no início dos anos 2000, pela empresa Objet Geometries Ltd (Israel). O nome vem da abreviatura de Photopolymer Jetting, que consiste em utilizar um sistema tipo jato de tinta para depositar a resina em pequenas gotas sobre uma base elevatória e imediatamente após a deposição, lançar uma luz Ultravioleta para a cura da camada (UDROIU; BRAGA, 2017). O sistema PolyJet apresenta bom acabamento, com ótima precisão apresentando velocidade de impressão alta, porém, após o processo é necessária uma limpeza, desperdíçando material (ABREU, 2015).

Como descrito por Reis (2016), o método ZPrinter, conhecido também como processo de TDP (Three dimensional Printing), utiliza-se de pó cerâmico ou polimérico, onde uma plataforma que se desloca sobre um eixo $z$ depositando e regularizando as camadas de pó a serem aglutinados, e com a cabeça de impressão abastece um liquido aglutinante, solidificando o material. Tal processo possibilita fabricar objetos com diferentes cores, de acordo com a cor do pó, porém apresenta baixa precisão e material frágil, além de ser um sistema com alto custo.

Por último, o método por Extrusão Plástico, ou deposição de material fundido, sendo o mais utilizado. O processo de fabricação do objeto consiste em aquecer o material (filamento plástico), até uma temperatura acima da temperatura de fusão, transformando o material em fluido para extrusão, que se solidifique assim que entre em contato com uma superfície mais fria. O processo é repetido camada por camada até completar o modelo ou peça (REIS, 2016).

O Extrusão Plástico é o mais lento entre os métodos citados, podendo o tipo filamento utilizado variar a qualidade da peça, assim como ela pode sofrer algum defeito por causa da variação de temperatura. Mas como vantagem principal é a capacidade de produzir peças funcionais e resistentes, e reproduzir modelos mais baratos, por exemplo, as impressoras RepRaps (ABREU, 2015).

\section{Aplicação das Impressoras 3D}

Se faz necessário comentar de qual forma a impressão 3D vem sendo utilizada dentro de vários sistemas industriais. Por ser um processo relativamente novo, encontra-se em fase de adoção por diferentes setores do mercado mundial, com especificidades para cada um, em uma revisão breve, serão abordados quais 
setores utilizam atualmente e quais passíveis de uso futuramente.

Gomes et al. (2017) afirmam que a indústria aeroespacial utiliza este serviço principalmente para prototipagem e desenvolvimento de tecnologia em menor escala, produzindo peças de pequeno porte, ferramentas, etc, desenvolvendo a tecnologia para uso de veículos não tripulados e partes maiores de foguetes como asas e motores.

No setor de alimentos se iniciou um processo de utilização da impressão 3D na fabricação de formas exclusivas para doces e talheres (MAGALHÃES; NASAJON, 2016). Também vem sendo produzidas através de impressoras 3D, comidas simples como pizza, brigadeiros e existe ainda em desenvolvimento, pesquisas e aprimoramentos de uma tecnologia para imprimir alimentos mais complexos (SANTOS, 2016).

De acordo com Gao et al. (2015) no setor de arquitetura e construção civil, impressoras 3D têm sido utilizadas para o desenvolvimento de maquetes e protótipos, inclusive há o desenvolvimento de impressoras 3D para a construção em escala real, sendo um dos setores que mais tem desenvolvido materiais e tecnologias para adoção na produção. A produção de mobiliário também tem utilizado a impressão 3D no desenvolvimento de móveis com conectores feitos através de técnicas de manufatura aditiva (DIGIANDOMENICO et al., 2017).

$\mathrm{Na}$ indústria automobilística como afirma Santos (2016), o acesso à tecnologia de impressão 3D proporciona o uso para o incremento da prototipagem dos modelos em desenvolvimento, ferramentas exclusivas, e num futuro próximo a produção de peças sobressalentes.

No setor de saúde, existe em desenvolvimento um extenso uso da manufatura aditiva no desenvolvimento de próteses personalizadas, implantes dentários (BUDZIK et al., 2016). Também modelos para aprendizagem de alunos e pacientes, que reproduzem através da impressão 3D, protótipos de partes do corpo humano para serem utilizados em aulas (MARTINS et al, 2017).

De acordo com Baden et al. (2015) existem modelos para impressão de equipamentos de laboratórios, disponíveis em sites de compartilhamento, sendo possível imprimir e montar partes de plástico juntamente com placas eletrônicas como Arduino, tornando-se possível construir um laboratório de baixo custo. $\mathrm{O}$ estado da arte de impressão em 3D na área de medicina vem desenvolvendo tecnologia para em breve poder imprimir órgãos artifiicias e partes do corpo humano (SANTOS, 2016).

No setor agrícola, estão aumentando estudos e aplicações de impressão 3D, sendo que algumas fábricas de implementos e máquinas utilizando tais técnicas na fabricação de protótipos de pequenas peças, encontrando assim pouca literatura e experimentos sobre o tema. Porém utilizando o mesmo método cartesiano de movimentação com eixos $\mathrm{X}, \mathrm{Y}, \mathrm{Z}$ e com sensores parecidos se desenvolveu um robô, que seria uma versão em escala maior de uma impressora 3D, para irrigação de plantas em estufas para produção na agricultura familiar (BATISTA et al., 2017).

A tecnologia está sendo cada vez mais utilizada na agricultura, mudando a indústria agrícola, utilizando a impressão 3D para criar protótipos e peças funcionais e o uso da Fabricação Aditiva diretamente por fazendeiros, podendo imprimir novas peças de reposição diretamente na fazenda, on-demand, comprando apenas o projeto de design do fabricante, deixando de esperar a entrega do produto (SCOTT, 2016).

Dessa forma, surge uma nova concepção de novos serviços digitais, a 
expansão de ofertas de produtos existentes em plataformas digitais ou o uso de tecnologias para reduzir custos em todos os níveis da própria cadeia produtiva (BRAUN et al., 2018).

Assim, a tecnologia de impressão 3D vem crescendo a cada ano, sendo utilizada em diversos setores como foi visto anteriormente e contribuindo para mudanças em modelos de produção e serviços, demonstrando a versatilidade de tal técnica e suas vantagens e perpectivas para 0 uso em inúmeros setores futuramente, inclusive o agrícola, seja nas indústrias de maquinários ou no campo com o produtor tendo a oportunidade de fabricar peças únicas ou de reposição para seu processo produtivo, demonstrando assim a capacidade de impacto no modelo de produção atual, principalmente no meio agrícola.

Posto isto, as aplicações para o meio rural são diversas, podendo ser fabricados partes de bicos de pulverização, partes de aspersores, objetos para uso na produção animal, tudo a curto prazo e com a possibilidade de serem fabricados na própria propriedade rual, visando o suporte nas atividades do agronegócio, tanto para reposição de peças, quanto também para início da atividade agropecuária, reduzindo o custo de um projeto ao produtor rural.

\section{CONSIDERAÇÕES FINAIS}

Dadas as profundas transformações no meio rural nos últimos anos, onde processos produtivos buscam em novas tecnologias melhorar a produtividade diminuindo o dispêndio de tempo, energia e capital, a prototipagem 3D se enquadra nos métodos de inovação capazes de alcançar tais resultados.

No caso do setor agrícola torna-se possível criar um serviço de reposição de peças mecânicas em lugares distantes à peças para impressão, diminuindo o dispêndio de tempo no processo de aquisição. Existe também a possibilidade de se criar peças que não são mais fabricadas, dificultando o sucateamento de maquinários em diversos processos produtivos.

Com a possibilidade de se produzir protótipos a custos e tempo menores que os atuais, a impressão 3D possibilita o desenvolvimento de outras tecnologias a baixo custo, otimizando o acesso da agricultura familiar a recursos que apenas grandes produtores possuem.

É uma área que vem emergindo a cada ano, sendo apontada por especialistas entre as principais tecnologias emergentes, necessitando assim de pesquisas que possam ampliar o conhecimento e a utilização na produção agrícola.

É possível propor a constituição de associações de produtores com máquinas de prototipagem 3D para uso conjunto no desenvolvimento de peças conforme suas necessidades e direcionadas a seus modelos produtivos, de forma totalmente customizada, ou o surgimento de empresas especializadas em otimização na produção agrícola que realizem serviços personalizados utilizando impressoras 3D.

\section{AGRADECIMENTOS}

Os autores agradecem à CAPES e CNPq pelo apoio financeiro para a concretização desta pesquisa.

\section{REFERÊNCIAS}

ABREU, S. A. C. Impressão 3D baixo custo versus impressão em equipamentos de elevado custo. Tese de Mestrado, Universidade do Porto, Protugal, 2015. 
BADEN, T.; CHAGAS, A. M.; GAGE, G.; MARZULLO, T.; PRIETO-GODINO, L. L.; \& EULER, T. Open Labware: 3-D printing your own lab equipment. PLoS biology. v. 13, n. 3, p. e1002086, 2015. Disponível em: <https://doi.org/10.1371/journal.pbio.1002175 >. doi: 10.1371/journal.pbio.1002175.

BAYGIN, M.; YETIS, H.; KARAKOSE, M.; \& AKIN, E. An effect analysis of industry 4.0 to higher education. In: Information Technology Based Higher Education and Training (ITHET), 2016 15th International Conference on. IEEE. p. 1-4, 2016. Disponível em: <https://doi.org/10.1109/ITHET.2016.7760744 >. doi: 10.1109/ITHET.2016.7760744.

BATISTA, A. V. D. A.; ALBIERO, D.; VIANA, T. V. D. A.; MONTEIRO, L. D. A.; CHIODEROLI, C. A., SOUSA, I. R. S. D., \& AZEVEDO, B. M. Multifunctional Robot at low cost for small farms. Revista Ciência Rural. v. 47, n. 7, 2017. Disponível em: <http://dx.doi.org/10.1590/0103-8478cr20170080>. doi: 10.1590/0103$8478 \mathrm{cr} 20170080$.

BERMAN, B. 3-D printing: The new industrial revolution. Business horizons, v. 55, n. 2,2012 p. 155-162, Disponível em: <https://doi.org/10.1016/j.bushor.2011.11.003>. doi: 10.1016/j.bushor.2011.11.003.

BRASIL, Governo. Plano Setorial de Mitigação e de Adaptação às Mudanças Climáticas para a Consolidação de uma Economia de Baixa Emissão de Carbono na Agricultura. Plano ABC (Agricultura de Baixa Emissão de Carbono). Coordenado por Casa Civil da Presidência da República, Ministério da Agricultura, Pecuária e Abastecimento (MAPA) e Ministério do Desenvolvimento Agrário (MDA). Versão final-13/01, 2012.

BRAUN, A.; COLANGELO, E.; STECKEL, T. Farming in the Era of Industrie 4.0. 2018. Disponível em: <https://doi.org/10.1016/j.procir.2018.03.176>. doi: 10.1016/j.procir.2018.03.176.

BUDZIK, G.; BUREK, J.; BAZAN, A.; \& TUREK, P. Analysis of the accuracy of reconstructed two teeth models manufactured using the 3DP and FDM technologies. Strojniški vestnik-Journal of Mechanical Engineering. v. 62, n. 1, p. 11-20, 2016. Disponível em: <https://doi.org/10.5545/sv-jme.2015.2699>. doi: 10.5545/svjme.2015.2699.

CONNER, B. P.; MANOGHARAN, G. P.; MARTOF, A. N.; RODOMSKY, L. M.; RODOMSKY, C. M.; JORDAN, D. C.; \& LIMPEROS, J. W. Making sense of 3-D printing: Creating a map of additive manufacturing products and services. Additive Manufacturing. v. 1, p. 64-76, 2014. Disponível em: <https://doi.org/10.1016/j.addma.2014.08.005>. doi: 10.1016/j.addma.2014.08.005.

DA COSTA, C. Indústria 4.0: o futuro da indústria nacional. POSGERE-PósGraduação em Revista/IFSP-Campus São Paulo, v. 1, n. 4, p. 5-14, 2017.

DA SILVA, M. C.; MASSARU BLOK MOTTER, V.; \& FELICE, F. A UTILIZAÇÃO DE UM SISTEMA COMPUTACIONAL DE BAIXO CUSTO PARA A CONFECÇÃO DE 
PLACAS DE CIRCUITO IMPRESSO. Anais: EVINCI-UniBrasil, v.3, n.1, 217-217, 2018.

DIGIANDOMENICO D.; LANDIM, G.; FISCHER, H. Trançado: computational design thinking applied to a urban furniture project. Gestão \& Tecnologia de Projetos. São Paulo - SP. v. 12, n. 3, p. 47-58, 2017. Disponível em: <https://doi.org/10.11606/gtp.v12i3.134232>. doi: 10.11606/gtp.v12i3.134232.

EMBRAPA. Visão 2014-2034: O futuro do desenvolvimento tecnológico da agricultura brasileira. Brasília, DF: Embrapa, 2014. Disponível em: $<$ https://www.embrapa.br/documents/1024963/1658076/O+Futuro+de+Desenvolvime nto+Tecnol\%C3\%B3gico+da+Agricultura+Brasileira++s\%C3\%ADntese.pdf/ddb0a14 7-234d-47f1- 8965-1959ef82311d>. Acesso em: 10 ago. de 2017.

FORNAZIER, A.; VIEIRA FILHO, J. E. R. Heterogeneidade estrutural no setor agropecuário brasileiro: evidências a partir do censo agropecuário de 2006. Brasília: Ipea, 2012. (Texto para Discussão, n. 1708).

GAO, W.; ZHANG, Y.; RAMANUJAN, D.; RAMANI, K.; CHEN, Y.; WILLIAMS, C. B.; \& ZAVATTIERI, P. D. The status, challenges, and future of additive manufacturing in engineering. Computer-Aided Design. v. 69, p. 65-89, 2015. Disponível em:<https://doi.org/10.1016/j.cad.2015.04.001>. doi: 10.1016/j.cad.2015.04.001.

GOMES, R. R.; DE FIGUEIREDO, H. V.; MENDES, A. C.; \& MUELLA, M. T. D. A. H. Análise, Modelagem e Impressão 3D de um Frame para Vants do Tipo Quadrícopteros para Voos em Ambiente Indoor. Revista Univap. v. 22, n. 40, p. 414, 2017. Disponível em: <http://dx.doi.org/10.18066/revistaunivap.v22i40.944>. doi: $10.18066 /$ revistaunivap.v22i40.944.

HAUSMAN, K. K.; HORNE, R. 3D Printing For Dummies. Hoboken, New Jersey: John Wiley \& Sons. 384 p, 2014.

HD STORE. Tipos de impressora 3D: conheça as mais importantes. Blog HD Store. 2017. Disponível em: <https://blog.hdstore.com.br>. Acesso em: 19 set. 2018.

HUBA, M.; KOZÁK, Š. From E-learning to Industry 4.0. In: Emerging eLearning Technologies and Applications (ICETA), 2016 International Conference on. IEEE. p. 103-108, 2016.

JIANG, H.; ZHAO, S.; YUAN, Y.; ZHANG, L.; DUAN, L.; \& ZHANG, W. The coupling relationship between standard development and technology advancement: A game theoretical perspective. Technological Forecasting and Social Change, 2017. Disponível em: <https://doi.org/10.1016/j.techfore.2017.11.018>. doi: 10.1016/j.techfore.2017.11.018.

JONES, R.; HAUFE, P.; SELLS, E.; IRAVANI, P.; OLLIVER, V.; PALMER, C.; BOWYER, A. RepRap-the replicating rapid prototyper. Robotica. v. 29, n. 01, p. 177-191, 2011. Disponível em: <https://doi.org/10.1017/S026357471000069X>. doi: $10.1017 /$ S026357471000069X. 
KALSOOM, U.; NESTERENKO, P. N.; PAULL, B. Current and future impact of 3D printing on the separation sciences. TrAC Trends in Analytical Chemistry, 2018. Disponível em: <https://doi.org/10.1016/j.trac.2018.06.006>. doi: 10.1016/j.trac.2018.06.006.

KAPETANIOU, C.; RIEPLE, A.; PILKINGTON, A.; FRANDSEN, T.; \& PISANO, P. Building the layers of a new manufacturing taxonomy: How 3D printing is creating a new landscape of production eco-systems and competitive dynamics. Technological Forecasting and Social Change, 2018, 128: p.22-35, 2018. Disponível em: <https://doi.org/10.1016/j.techfore.2017.10.011>. 10.1016/j.techfore.2017.10.011.

KIETZMANN, J.; PITT, L.; BERTHON, P. Disruptions, decisions, and destinations: Enter the age of 3-D printing and additive manufacturing. Business Horizons. v. 58, n. 2, p. 209-215, 2015. Disponível em: <https://doi.org/10.1016/j.bushor.2014.11.005>. doi: 10.1016/j.bushor.2014.11.005.

LOW, Z. X.; CHUA, Y. T.; RAY, B. M.; MATTIA, D.; METCALFE, I. S.; \& PATTERSON, D. A. Perspective on 3D printing of separation membranes and comparison to related unconventional fabrication techniques. Journal of Membrane Science, v. 523, p. 596-613, 2017. Disponível em: <https://doi.org/10.1016/j.memsci.2016.10.006>. doi: 10.1016/j.memsci.2016.10.006.

MARTINS, K. Y. N.; BRANCO, R. R. C.; DE ANDRADE, K. D.; DA SILVA, J. B.; \& DE OLIVEIRA, M. D. L. F. Desenvolvimento de um Educador Vaginal Através do Processo de Manufatura Aditiva (Impressão 3D). Revista Pesquisa em Fisioterapia. v. 7, n. 1, 2017. Disponível em: <http://dx.doi.org/10.17267/22382704rpf.v7i1.1239>. doi: 10.17267/2238-2704rpf.v7i1.1239.

MAGALHÃES, C. F.; NASAJON, D. R. BIKO: Talher Descartável para Pequenas Porções de Alimentos. Blucher Design Proceedings, v. 2, n. 9, p. 5362-5373, 2016.

MENDONÇA, M. G.; LEHFELD, L. S. Agricultura sustentável: agroecologica sob o enfoque da bioética. In: Anais. Congresso Brasileiro de Processo Coletivo e Cidadania. p. 155-160, 2015.

NIKANDER, J. Suitability of papiNet-standard for straw biomass logistics. Journal of Industrial Information Integration, v. 6, p.11-21, 2017. Disponível em: $<$ https://doi.org/10.1016/j.jii.2017.04.004>. doi: 10.1016/j.jii.2017.04.004.

RAMOS, V. D. C. Estratégia empresarial e dinâmicas de inovação: referências para uma gestão estratégica de sucesso no setor vitivinícola brasileiro. Tese (Doutorado em Gestão) - Universidade de Évora, Portugal, 304p. 2015.

REDIVO, A. R.; TRÊS, CACIO; FERREIRA, GERALDO ALVES. A Tecnologia de Informação aplicada ao Agronegócio: Um estudo sobre o "Sistema Agrogestor" nas fazendas do Município de Sinop/MT. Revista Contabilidade \& Amazônia. v. 1, n. 1, p. 43-52, 2012. 
REIS, J. F. S. Desenvolvimento e projeto de impressora DMF para impressão multicolor. Dissertação de Mestrado. Universidade de Aveiro. Portugal, 132p. 2016.

SANTOS, R. C.; SUAREZ, A. H. T.; SOUZA, C. M. A.; JUNQUEIRA, M. H. Uso de planilha eletrônica para determinação do risco ao estresse climático de suínos e frangos de corte. In: Rosalen, D.L.; Furlani, C.E.A.; Faria, R.T. (Org.). Jubileu de Ouro da SBEA. 1ed.Jaboticabal: Associação Brasileira de Engenharia Agrícola. v. 1, p. 8-16, 2015.

SANTOS, S. L. B. Impressão 3D: perspectivas de adoção na Indústria Portuguesa. Tese de Doutorado, Universidade Nova Lisboa, Portugal. 72p. 2016.

SCHWAB, K. The fourth industrial revolution. Crown Business, 194p. 2017.

SCOTT, Clare. 3D Printing and Other High-Tech Solutions Could Create Lots of New Jobs in Agriculture. Disponível em: <https://3dprint.com/115833/3d-printingagriculturejobs/>.Acesso em: 20 set. 2018.

UDROIU, R.; BRAGA, I. C. Polyjet technology applications for rapid tooling. In: MATEC Web of Conferences. EDP Sciences. p. 03011, 2017. Disponível em: <https://doi.org/10.1051/matecconf/201711203011>.

doi: 10.1051/matecconf/201711203011.

ZUCCA, R.; VIEIRA NETO, J. G.; SANTOS, R. C.; LOVATTO, J.; COSTA, M. V. Uso de resíduos de construção como agregado graúdo destinado à confecção de blocos para alvenaria de vedação. Enciclopédia Biosfera, v. 15, p. 1381-1392, 2018. Disponível em: <https://doi.org/10.18677/EnciBio_2018A119>. doi: 10.18677/EnciBio_2018A119.

ZYLBERSZTAJN, D.; NEVES, M. F. \& CALEMAN, S. M. D. Q. (Org.). Gestão de Sistemas de Agronegócios. São Paulo: Atlas, 2014.

WEF - WORLD ECONOMIC FORUM. "Deep Shift Technology Tipping Points and Societal Impact," 2015. [Online]. Disponível em: <http://www3.weforum.org/docs/WEF_GAC15_Technological_Tipping_Points_report _2015.pdf>. Acesso em: 24 ago. 2018.

WOHLERS, T. 3D printing and additive manufacturing state of the industry. Annual Worldwide Progress Report. Wohlers Associates, 2014.

WOHLERS, T. 3D printing and additive manufacturing state of the industry. Annual Worldwide Progress Report. Wohlers Associates, 2016.

WOHLERS, T. 3D Printing and Additive manufacturing state of the industry. Anual Worldwide Progress Report. Wohlers Associates, 2017. 